\title{
On Postpartum Depression, Hormonal Problems, and Practice Management for Medical Home Implementation
}

Within the constraints of the need for rapid dissemination of new data, we like publishing related articles together. This issue we have collected articles related to 3 topic areas: postpartum depression, hormonal problems in family medicine, and implementation of aspects of the medical home. In addition, from Dr. Pathman, a member of the 7 ABFM Board of Editors, we have excellent information about the National Health Service Corps and its recent expansion, which will increase the availability of primary care for the underserved.

\section{Postpartum Depression}

The topic of postpartum depression (PPD) is particularly pertinent because $\mathcal{F} A B F M$ articles on this topic are consistently among the most-read papers. (We know this because we get reports about how often individual articles are downloaded from our Website.) In this issue, we have 5 articles relating to postpartum issues, 4 of which are specific to depression: (1) one comparing 2 questionnaires for potential use in case detection ${ }^{1}$; (2) one discussing a method to train providers to recognize PPD, which is available to all our readers through the internet ${ }^{2}$; (3) one outlining a stepped care approach for treatment $^{3}$; and (4) a pilot investigation into whether or not PPD is affected by the seasons. ${ }^{4}$ Two have lead authors who are among the best known PPD researchers, Drs. Dwenda Gjerdingen and Barbara Yawn, both of whom are family physicians and funded by National Institutes of Health.

In the Yawn et al article, ${ }^{1}$ the Edinburgh Postnatal Depression Scale and the 9-item Patient Health Questionnaire were compared as screening tools for PPD. A couple of very good results were that the women readily completed either questionnaire with few unanswered questions, and the women also readily completed the suicide questions. The data suggest that the Edinburgh Post-

Conflict of interest: The authors are editors of the $7 A B F M$. natal Depression Scale may have the advantage of fewer callbacks and the PHQ-9 may require follow-up of more patients, the clinical utility of which is unknown. If the PHQ-9 is the general routine in a family medicine office, it would seem reasonable to continue to use it across patients rather than switch to another instrument just for postpartum patients because simplicity often means greater success for these types of practice activities. In their study, Gjerdingen et $\mathrm{al}^{3}$ found that the collaborative stepped care intervention was associated with higher recognition of depression and greater initiation of treatment. Possibly, the most intriguing finding was the high rate of self-reported (those not diagnosed by the research interview) depression; those self-reporting this depression had negative outcomes at 9 months. Baker et $\mathrm{al}^{2}$ provides a free, open-access, Internet-based training program (also funded by the National Institutes of Health) with a name interestingly similar to the title of the paper by Gjerdingen et al: "Support and Training to Enhance Primary Care for Postpartum Depression" (www.step-ppd.com). Further, given that a significant number of people are affected by Seasonal Affective Disorder, Panthangi et $\mathrm{al}^{4}$ wondered if there would be seasonal variation in the rate of PPD. Their pilot study in 2 family medicine offices and one obstetrics/gynecology office did not find statistically significant results but there were tantalizing hints, particularly that PPD was lowest in the summer; these results suggest a large study should be undertaken.

In our final article in this issue about postpartum issues, Clinch et $\mathrm{al}^{5}$ found that $60 \%$ of women reported discussions with a health care provider about returning to work after delivery, and these were mostly initiated by the patients, not the providers. Most women found the discussions helpful, some substantially so, and some women without such a discussion wish they had had one. The content of such discussions should include consider- 
ations of the health of both the mother and the infant.

\section{Hormonal Problems in Family Medicine}

We have 8 articles about hormone-related problems. Five of these articles are about that exploding endocrinologic disease, diabetes. ${ }^{6-10}$ Crawford et $\mathrm{al}^{6}$ report on positive results from the largest randomized, controlled trial to date of the use of overthe-counter cinnamon in patients with uncontrolled diabetes. The amount of reduction in glycosylated hemoglobin $(-0.83 ; 95 \%$ CI, $0.46-$ 1.20), was similar to what has been seen in some prescription medication trials, yet cinnamon seems safer, definitely cheaper, and requires fewer capsules than what is often needed for other complementary agents (only 2 150-mg capsules daily). Unfortunately, there was no ability to blind the capsules, so it is not a truly placebo-controlled trial.

Previous studies suggest that inadequate intensification of treatment during individual visits is associated with worse control of diabetes, but the decisions behind this are poorly understood. Thus, Grant et $\mathrm{al}^{7}$ sensibly used a clinical vignette (via video) to help physicians decide whether or not to step up treatment in a patient with diabetes and understand borderline indications for intensified treatment. The unsurprising results: those who did not intensify treatment wanted more clinical data and those who did intensify treatment were more worried about complications. The surprising results: those who intensified treatment more frequently noted other patient-based concerns, such as the cost of medications and adherence. Finally, the gratifying results: none or very little of the differences were based on patients' socioeconomic characteristics.

In translating research into practice, Matvienko et $\mathrm{al}^{8}$ had graduate students be the interventionists to implement the lifestyle intervention program called the Diabetes Prevention Program, which is famous for delaying or preventing diabetes. ${ }^{11}$ The results indicate that this type of program could be implemented in the community with results similar to the major research trial. We think family physicians also can delay or prevent diabetes in practice with repetitive, short interventions over a sustained period of time to help patients improve their lifestyles (ie, nutrition and exertion).

Diaz et $\mathrm{al}^{9}$ use the population-based National Health and Nutrition Examination Survey data to illustrate that reducing vitamin D deficiency might reduce diabetes-associated nephropathy. Given the higher rate of vitamin $\mathrm{D}$ deficiency among minorities, this finding supports the notion that vitamin $\mathrm{D}$ deficiency is one reason for the higher rates of nephropathy in minorities with diabetes. As reviewed by Saliba, ${ }^{12}$ vitamin $\mathrm{D}$ is a known and major treatment for the secondary hyperparathyroidism of nephropathy. It seems that it is time for a formal, randomized, controlled trial of vitamin $\mathrm{D}$ in diabetic nephropathy before hyperparathyroidism sets in, and the investigators should be sure to include sufficient minority patients to identify any differential impact.

Among people with diabetes, another common deficiency is that of vitamin $\mathrm{B}_{12}$, as shown by Pflipsen et al. ${ }^{10}$ Metformin is one of the causes, and the data in this article suggests that a multivitamin is a simple, cheap, and effective method for preventing many cases of this deficiency.

Waring et $\mathrm{al}^{13}$ found that documentation of obesity was greater with increasing obesity, as were obesity-associated interventions, particularly when there were comorbidities. This is understandable, but we probably need to start documentation sooner, when patients have a lower body mass index, to prevent further expanding waist lines.

Postmenopausal hormone therapy presents many challenges for clinicians and their patients. Getting sufficient long-term data about the multiple different medications over a significant length of time in a generally healthy population has been part of the difficulty. Langer et $\mathrm{al}^{14}$ review what we know about low-dose therapy. Several current national recommendations suggest low-dose therapy for as little time as necessary for symptoms of menopause.

\section{Practice Management for the Medical Home}

We have 2 articles related to practice management aspects pertinent to implementing medical homes: satisfaction with use of electronic health records in the office setting ${ }^{15}$ and the successful use of telephonic case management to help special needs populations become attached to a primary care physician. ${ }^{16}$

In the next issue, our article themes will be infectious diseases in family medicine, screening, and more articles pertinent to the medical home.

Marjorie A. Bowman, MD, MPA Anne Victoria Neale, PhD, MPH 


\section{References}

1. Yawn BP, Pace W, Wollan PC, et al. Concordance of Edinburgh Postnatal Depression Scale (EPDS) and Patient Health Questionnaire (PHQ-9) to assess increased risk of depression among postpartum women. J Am Board Fam Med 2009;22:483-91.

2. Baker CD, Kamke H, O'Hara MW, Stuart S. Webbased training for implementing evidence-based management of postpartum depression. J Am Board Fam Med 2009;22:588-9.

3. Gjerdingen D, Crow S, McGovern P, Miner M, Center B. Stepped care treatment of postpartum depression: impact on treatment, health, and work outcomes. J Am Board Fam Med 2009;22:473-82.

4. Panthangi V, West P, Savoy-Moore RT, Geeta M, Reickert E. Is seasonal variation another risk factor for postpartum depression? J Am Board Fam Med 2009;22:492-7.

5. Clinch CR, Grzywacz JG, Tucker J, Walls JK, Arcury TA. Characteristics of mother-provider interactions surrounding postpartum return to work. J Am Board Fam Med 2009;22:498-506.

6. Crawford P. Effectiveness of cinnamon for lowering hemoglobin $\mathrm{A} 1 \mathrm{C}$ in patients with type 2 diabetes: a randomized, controlled trial. J Am Board Fam Med 2009;22:507-12.

7. Grant RW, Lutfey KE, Gerstenberger E, Link CL, Marceau LD, McKinlay JB. The decision to intensify therapy in patients with type 2 diabetes: results from an experiment using a clinical case vignette. J Am Board Fam Med 2009;22:513-20.

8. Matvienko OA, Hoehns JD. A lifestyle intervention study in patients with diabetes or impaired glucose tolerance: translation of a research intervention into practice. J Am Board Fam Med 2009;22:535-43.

9. Diaz VA, Mainous AG, Carek PJ, Wessell AM, Everett CJ. The association of vitamin D deficiency and insufficiency with diabetic nephropathy: implications for health disparities. J Am Board Fam Med 2009;22:521-7.

10. Pflipsen MC, Oh RC, Saguil A, Seehusen DA, Topolski R. The prevalence of vitamin B12 deficiency in patients with type 2 diabetes: a cross sectional study. J Am Board Fam Med 2009;22:528534.

11. Knowler WC, Barrett-Connor E, Fowler SE, et al. Diabetes Prevention Program Research Group. Reduction in the incidence of type 2 diabetes with lifestyle intervention or metformin. N Engl J Med 2002;346:393-403.

12. Saliba W, El-Haddad B. Secondary hyperparathyroidism: pathophysiology and treatment. J Am Board Fam Med 2009;22:574-81.

13. Waring ME, Roberts MB, Parker DR, Eaton CB. Documentation and management of overweight and obesity in primary care. J Am Board Fam Med 2009; 22:544-52.

14. Langer RD. Efficacy, safety, and tolerability of lowdose hormone therapy in managing menopausal symptoms. J Am Board Fam Med 2009;22:563-73.

15. Irani JS, Middleton JL, Marfatia R, Omana ET, D'Amico F. The use of electronic health records in the exam room and patient satisfaction: a systematic review. J Am Board Fam Med 2009;22:553-62.

16. Kahn LS, Aiello J, Berdine DE, Fox CH. The use of telephonic case management to link a special-needs population with a primary care physician. J Am Board Fam Med 2009;22:585-7. 